Int J Hyg Environ Health. 2019 June ; 222(5): 778-789. doi:10.1016/j.jhheh.2019.05.002.

\title{
Urinary Metals and Metal Mixtures in Midlife Women: the Study of Women's Health Across the Nation (SWAN)
}

\author{
Xin Wang ${ }^{\mathrm{a}}$, Bhramar Mukherjee ${ }^{\mathrm{b}}$, Stuart Batterman ${ }^{\mathrm{c}}$, Siobán D. Harlow ${ }^{\mathrm{a}}$, and Sung Kyun \\ Park $^{\mathrm{a}, \mathrm{c}}$ \\ aDepartment of Epidemiology, School of Public Health, University of Michigan, Ann Arbor, MI \\ bDepartment of Biostatistics, School of Public Health, University of Michigan, Ann Arbor, MI \\ 'Department of Environmental Health Sciences, School of Public Health, University of Michigan, \\ Ann Arbor, MI
}

\section{Abstract}

Background: Little is known about the extent of exposure to metals and metal mixtures among midlife women.

Objectives: We assessed exposure to multiple metals in the Study of Women's Health Across the Nation (SWAN), a multi-site, multi-racial/ethnic cohort of women at midlife.

Methods: We measured urinary concentrations of 21 metals (arsenic, barium, beryllium, cadmium, cobalt, chromium, cesium, copper, mercury, manganese, molybdenum, nickel, lead, platinum, antimony, tin, thallium, uranium, vanadium, tungsten and zinc) using high-resolution inductively coupled plasma-mass spectrometry among 1,335 white, black, Chinese and Japanese women aged 45-56 years at the third SWAN annual visit (1999-2000). Least squared geometric mean concentrations were compared across race/ethnicity, education, financial hardship, smoking, secondhand smoking, seafood intake and rice intake groups. Overall exposure patterns of multiple metals were derived using k-means clustering method.

Results: The percentage of women with detectable concentrations of metals ranged from $100 \%$ for arsenic, cesium, molybdenuMAnd zinc, to less than 5\% for platinum; 15 metals had detection rates of $70 \%$ or more. Asian women, both Chinese and Japanese, had higher urinary concentrations of arsenic, cadmium, copper, mercury, molybdenum, lead and thallium, compared with other race/ethnic groups, independent of sociodemographic, lifestyle, dietary, and geographic characteristics. Seafood and rice intake were important determinants of urinary arsenic, cesium, mercury, molybdenum and lead levels. Two distinct overall exposure patterns- "high" vs. "low" -were identified. Women in the "high" overall exposure pattern were more likely to be Asians,

Corresponding author: Sung Kyun Park, Department of Epidemiology, University of Michigan, M5541 SPH II, 1415 Washington Heights, Ann Arbor, Michigan 48109-2029. Phone: (734) 9361719. sungkyun@umich.edu.

Publisher's Disclaimer: This is a PDF file of an unedited manuscript that has been accepted for publication. As a service to our customers we are providing this early version of the manuscript. The manuscript will undergo copyediting, typesetting, and review of the resulting proof before it is published in its final citable form. Please note that during the production process errors may be discovered which could affect the content, and all legal disclaimers that apply to the journal pertain.

Declaration of competing interests

The authors declare they have no actual or potential competing interest. 
current smokers, and to report high consumption of seafood and rice. Black women were less likely to have the high exposure pattern.

Conclusions: Metal exposure of midlife women differs by racial/ethnic, sociodemographic, lifestyle, dietary, and geographic characteristics. Asian women may be experiencing the highest exposures to multiple metals compared with other racial/ethnic groups in the United States.

\section{Graphical Abstract}
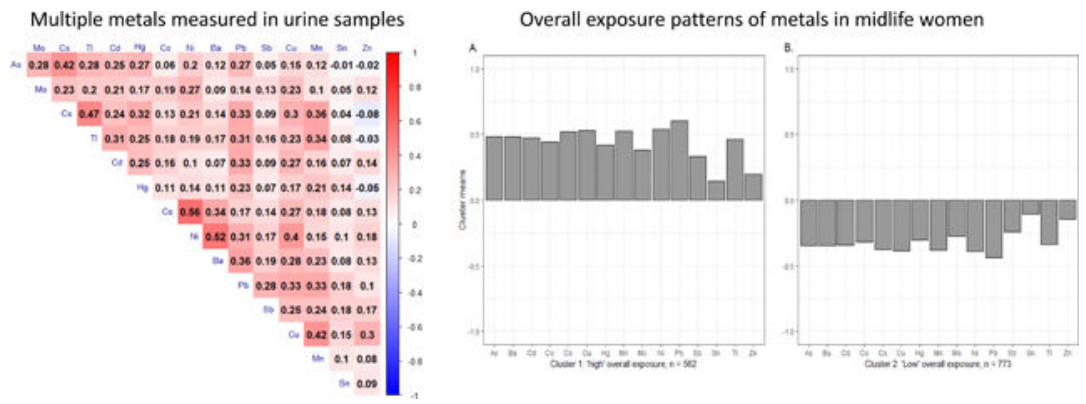

\section{Keywords}

Metals; Mixtures; Clustering analysis; Women; Mid-life

\section{INTRODUCTION}

Metals occur naturally in the environment and are widely used in industrial, agricultural, and manufacturing processes. Individuals are commonly exposed to metals found in soil, water, air, dust, food, and consumer products (Alloway, 2013; Bosch et al., 2016; M ohod and Dhote, 2013). Arsenic, cadmium, lead and mercury are among the most toxic environmental pollutants. Although a remarkable reduction in environmental sources of such toxic metals has been achieved in the United States (U.S.) over the last several decades (Calafat, 2012), low-to-moderate chronic exposure has been associated with numerous health outcomes, including cardiovascular diseases, kidney diseases, metabolic diseases, neurocognitive outcomes, some cancers, and mortality (Lanphear et al., 2018; Mohammed Abdul et al., 2015; Navas-Acien et al., 2007; Satarug et al., 2009; Zahir et al., 2005). Other metals, like cobalt, copper, manganese, molybdenum, vanadium and zinc, are necessary for multiple biochemical pathways and required for certain enzymes (Fraga, 2005). Given the role of essential elements in human nutrition, deficiencies are frequently associated with diseases; excessively high concentrations of such metals may also have toxic effects (Fraga, 2005).

Exposure to metals is unequally distributed, and this unequal distribution is often related to sociodemographic, lifestyle, dietary and geographic factors. For example, lead body burden varies across socioeconomic and racial/ethnic groups (Hu et al., 1996; Theppeang et al., 2008). Exposure to arsenic, lead, cadmium, mercury, cesium, thallium and antimony has been observed as a function of poverty-income-ratio among the U.S. adults (Tyrrell et al., 2013). Exposures to arsenic, cadmium, mercury and lead with has also been linked to lifestyle factors, such as smoking and food/dietary intake, especially seafood and rice 
(Castro-González and Méndez-Armenta, 2008; Gilbert-Diamond et al., 2011; Wang et al., 2017).

Few studies have evaluated exposures to metals in midlife women despite the growing appreciation of the importance of this lifestage to health and wellbeing. The menopausal transition is characterized by a shift in women's sex hormone profile owing to permanent changes in ovarian function which is associated with increased risk of chronic diseases, most notably cardio-metabolic disorders (S. R. Davis et al., 2012; Kim, 2012; Polotsky and Polotsky, 2010; Stuenkel, 2017). Exposure to toxic metals during this window of susceptibility may increase the risk of adverse health consequences associated with ovarian aging. For example, menopause has been suggested to play an important role in the mobilization of lead from bone into the circulation due to an increased bone turnover rate (Hernandez-Avila et al., 2000; Tsaih et al., 2001). Bone lead stores accrued from cumulative environmental exposures for decades are the major endogenous source of lead (Tsaih et al., 2001). Lead exposure has been associated with health outcomes such as hypertension and coronary heart disease (Ding et al., 2016; Korrick et al., 1999). To date, however, no study has examined midlife women's metal exposure profile comprehensively or identified characteristics of highly exposed subpopulations.

Exposures to different metals may be correlated due to common environmental sources or similarities in metabolic pathways (Pang et al., 2016). Given the complexity in the correlations, simultaneous exposure to multiple metals may result in effects that can depart from a simple summation of the effects of single metals (Park et al., 2017, 2014; Wang et al., 2018). Identification of overall exposure patterns is critical for evaluating the associations between metal mixtures and health outcomes. These issues have been recognized by the National Institute of Environmental Health Sciences (NIEHS), which has set understanding the field of exposures to mixtures of environmental chemicals as one of the goals of the 2018-2023 Strategic Plan (Birnbaum, 2018).

In this paper, we report on measurements of 21 metals in urine samples collected from the Study of Women's Health Across the Nation (SWAN), a multi-site, multi-race/ethnic cohort of women aged 45-56 years at the time of urine collection. The overall objectives were (1) to examine the distributions of urinary concentrations of metals, (2) to identify subgroups exposed to different patterns of metals using the k-means clustering method, a nonparametric clustering method seeking a minimum error sum of squares, which could suggest specific exposure patterns of metals (Jain, 2010), and (3) to evaluate associations of demographic, socioeconomic, lifestyle, dietary and geographical characteristics with each urinary metal, as well as with exposure patterns of multiple metals, in this diverse population of midlife women.

\section{METHODS}

\section{Study Population}

Women in the present study were participants in the SWAN, an ongoing, a multi-site, multiethnic, community-based longitudinal study of the natural history of menopause designed to address the effect of the menopausal transition on subsequent health and to identify risk 
factors for age-related chronic diseases (Sowers et al., 2000). Between 1996 and 1997, 3,302 women were enrolled from seven study sites, including Boston, MA; Chicago, IL; southeast Michigan, MI; Los Angeles, CA; Oakland, CA; Newark, NJ; and Pittsburgh, PA. Each site enrolled white women and women from one minority group (black women from Boston, Chicago, Southeast Michigan, and Pittsburgh; Chinese women from Oakland; Japanese women from Los Angeles; Hispanic women from Newark). Black, Chinese, Japanese, and Hispanic women comprised greater proportions of the SWAN population than their respective proportions in the general U.S population, reflecting the study design to oversample these groups (Sowers et al., 2000). Eligibility criteria for enrollment into the SWAN cohort included the following: age 42 to 52 years, intact uterus and at least one ovary, no use of exogenous hormones affecting ovarian function in the past 3 months, at least one menstrual period in the previous 3 months, and self-identification with a site's designated racial/ethnic groups. Institutional review board approval was obtained at each study site, and all participants provided signed informed consent at each study visit.

The SWAN Multi-Pollutant Study used urine samples from the SWAN Repository collected during the third SWAN follow-up visit (visit 03, 1999-2000) for environmental exposure assessment. A subset of 1,400 SWAN participants from the five SWAN sites who provided urine samples to the SWAN Repository (Boston, southeast Michigan, Los Angeles, Oakland and Pittsburgh) were assayed for metal concentration determinations. Women from Chicago and Newark were excluded because urine samples were not collected in these two sites. This subpopulation, by design, included self-identified white, black, Chinese, and Japanese but not Hispanic women who were recruited exclusively from Newark. Among these five sites, women for whom urine samples were not available were less educated and more likely to be current smokers or obese than women with available urine. For this analysis, we excluded 2 participants with insufficient urine samples such that one or more metal concentrations could not be determined, and 63 participants with missing information on core covariates (smoking, secondhand smoking, education, financial hardship, seafood intake, rice intake, and urinary creatinine concentrations), leaving 1,335 eligible participants (95.6\%) for analysis. When compared to the 65 excluded women, women eligible for this analysis were similar with respect to age and racial distributions. An overview of our sampling procedure is illustrated in Figure S1.

\section{Urinary Metals}

Urine specimens were collected prior to $11 \mathrm{am}$ in the morning. First morning voided urine was collected. Aliquoted specimens were frozen and stored in ultra-low freezers at $-80{ }^{\circ} \mathrm{C}$ until they were later analyzed for the metal content. All specimens were collected and stored in the SWAN Repository (http://swanrepository.com/) using a systematic protocol. A total of 21 metals including total arsenic, barium, beryllium, cadmium, cobalt, chromium, cesium, copper, mercury, manganese, molybdenum, nickel, lead, platinum, antimony, tin, thallium, uranium, vanadium, tungsten and zinc were measured in the urine samples. All the urinary metals were analyzed with high-resolution inductively coupled plasma-mass spectrometry (ICP-MS) (Thermo Scientific iCAP RQ, Waltham, MA) by the Applied Research Center of NSF International (Ann Arbor, Michigan), a part of the Michigan Children's Health Exposure Analysis Resource (M-CHEAR) Laboratory Hub. We used the CDC method 
3018.3 (CDC, 2012), with modifications for the expanded metals panel. All standards, quality controls $(\mathrm{QCs})$, blanks, rinse solution and urine samples were diluted 10-fold in a diluent consisting of $2 \% \mathrm{HNO}_{3}$ solution containing the internal standards and gold. The samples were analyzed in two analysis modes - standard (default) for the majority of the metals, and kinetic energy discrimination (KED) for vanadium, chromium, arsenic, molybdenum and cadmium. The following QC procedures was conducted in parallel with sample analyses: (a) second source standards and spike and surrogate reco veries were tested periodically; (b) linearity and drift checks were performed with each sample batch; and (c) metal internal standards were used on each sample. Each sample run contained a minimum of 4 calibration standards and a blank. The coefficients of variation were $2.4-34.8 \%$ for the low QC pools; $1.6-4.0 \%$ for the high QC pools; and $1.8-4.0 \%$ for the laboratory fortified blank. The limits of detection (LODs) of each metal were determined during the method validation by running a dilution matrix blank 10 times and then calculating the standard deviation of the instrument response. The limit of detection was then defined by calculating three times the standard deviation. Metal concentrations below their LODs were assigned the LOD divided by the square root of 2 .

\section{Covariates}

Sociodemographic factors including age, race/ethnicity, educational attainment, and financial hardship were assessed at the SWAN baseline examination (1996-1997). Race/ ethnicity was classified into self-identified white, black, Chinese, or Japanese. Education was categorized as graduated from high school or less, attended some college, and graduated from 4-year college or higher degree. Financial hardship was derived from the question "How hard is it for you to pay for the very basics like food, housing, medical care and heating?" with the 3-level response indicating "very hard", "somewhat hard", and "not hard at all" (Hall et al., 2008). Lifestyle variables including cigarette smoking and secondhand smoking at home, work, and other social settings were collected at SWAN visit 03 with a self-administered questionnaire. Cigarette smoking was categorized as never, former, or current smoking. Total person-hours of secondhand smoking exposure per week was calculated and categorized as 0 hour per week, less than 5 hours per week and more or equal than 5 hours per week. Dietary intake was collected at the SWAN baseline, using a detailed semi-quantitative food frequency questionnaire (FFQ) adopted from Block FFQ (Block et al., 1986). The 103-food item FFQ included 4 seafood items (fried fish/fish sandwich, tuna fish/tuna salad, shellfish, and other fish) and 1 rice item (rice/dishes made with rice). For analysis, weekly seafood intake was computed by summing the frequency of intake for the 4 fish items. To obtain comparable numbers of participants in each group of food intake, we categorized seafood intake into tertiles as less than 1 time per week, 1 to 1.9 times per week, and greater than or equal to 2 times per week. Rice intake was categorized into tertiles as less than 1.5 times per week, 1.5 to 3.4 times per week, and greater than or equal to 3.5 times per week. Total energy intake was obtained from the FFQ based on each food intake. Urine creatinine was determined by the Cobas Mira analyzer (Horiba ABX, Montpellier, France) at SWAN visit 03 as makers of urine dilution. 


\section{Statistical Analysis}

Means and percentages of participant characteristics were calculated and examined by race/ ethnic groups. Detection rate, geometric mean and distribution percentiles for both the volume-based $(\mu \mathrm{g} / \mathrm{L})$ and creatinine-adjusted $(\mu \mathrm{g} / \mathrm{g}$ creatinine) concentrations of each urinary metal were calculated. Pairwise Spearman correlations among urinary creatinineadjusted metal concentrations were calculated and presented via a correlation-matrix heat map. To compare metal exposure profiles in SWAN to the concentrations in the U.S. general population, median creatinine-adjusted concentrations of urinary metals in white and black women aged 45-56 years (the age range of SWAN women) from the National Health and Nutrition Examination Survey (NHANES) 1999-2000 were calculated. The complex survey design of NHANES was considered using the R 'survey' package. We were noTable to compare metal concentrations in Chinese and Japanese women between SWAN and NHANES due to the limited number of Asian Americans included in the NHANES 19992000 cycle (the category "Non-Hispanic Asian" was not available until NHANES survey cycle 2011-2012) (CDC/NCHS, 2018).

We used analysis of covariance (ANCOVA) to examine the influence of race/ethnicity, education, financial hardship, smoking, secondhand smoking, seafood and rice intake on each natural log-transformed urinary metal concentration, given the right-skewed concentration distributions. This model enabled us to compare the expected least square geometric mean (LSGM) metal concentrations for selected determinants (e.g., across race/ ethnic groups), which were adjusted statistically so that participants had comparable levels of all other covariates in the model. To control for potential confounding, age and study sites were adjusted in all models (Santoro et al., 2011). All models were adjusted for urine creatinine to account for variations in dilution in spot urine samples (Barr et al., 2005; O'Brien et al., 2016). When seafood intake and rice intake were included in the model, we also adjusted for total energy intake (Willett et al., 1997). We calculated LSGM metal concentrations across the study sites within white and black women respectively, to assess potential geographical differences in metal exposures. Geographical differences could not be evaluated within the Chinese or Japanese groups as they were sampled at only one site by design. We also calculated and compared the LSGM metal concentrations between white and Chinese women within the Oakland site, and between white and Japanese women within the Los Angeles site, to examine potential race/ethnic differences in metal exposures within these sites.

K-means clustering was implemented to identify subgroups of SWAN participants with different overall exposure patterns of urinary metals. K-means clustering is a commonly used nonparametic clustering method partitioning quantitative variables towards different centroids seeking a minimum total within-cluster variation (Jain, 2010). This approach creates a single variable with $\mathrm{k}$ categories as different clusters where participants within the same cluster are as similar as possible and participants from different clusters are as dissimilar as possible, in terms of the quantitative variables (i.e., urinary metal concentrations). The k-means algorithm (1) randomly selected $\mathrm{k}$ centroids in a space of urinary metals and assigned each participant to the closest centroid by minimizing the distance to the corresponding centroid (within-cluster sum of squares), and (2) updated the 
centroids as the average of all data points in a cluster and again assigned each participant to the closet centroid. Step (2) was iterated until the cluster assignments stopped changing (convergence). Each cluster represents a subpopulation with a specific metals exposure pattern. All log-transformed urinary metals were standardized to make variables comparable before the k-means analysis. The number of optimal clusters (k) was determined based on (1) cubic clustering criterion (Warren and Sarie, 1983); (2) Elbow method (plotting total within-cluster sum of square vs. num ber of clusters); and (3) average Silhouette method (Rousseeuw, 1987).

To identify those risk factors most strongly associated with exposure to individual metals, as well as to the overall exposure patterns, variable selection was performed using backward elimination with an initial model including race/ethnicity, education, financial hardship, smoking and secondhand smoking and a threshold of $\mathrm{p}<0.05$ for retaining the variables in linear regression for individual metals, and logistic regression for the overall k-means clustering exposure patterns, respectively. Age, study site, and total energy intake were forced into all models to control for confounding. Urinary creatinine was forced into all linear regression but not into the logistic regression models since the overall exposure patterns were derived based on the creatinine adjusted-metal concentrations. Regression analyses and k-means clustering were performed only for metals for which the detection rate was $\geq 70 \%$.

To examine analytical consistency and robustness of our findings, we substituted specific gravity for urinary creatinine to adjust for urine dilution in all regression analyses, as a sensitivity analysis. Specific gravity was measured using a handheld digital refractometer (ATAGO model PAL-10S, Tokyo, Japan) at SWAN visit 03. All analyses were conducted using R, version 3.4.0.

\section{RESULTS}

Table 1 presents participants' characteristics by racial groups and for the total study population. Participants had a mean age of 49.4 years, ranging from 45 to 56 years, which was not significantly different across the four racial groups $(P=0.12)$. There were significant differences in education, financial hardship, smoking, secondhand smoking, seafood and rice intake between race/ethnic groups $(P S<0.001)$. Generally, black women reported the lowest socioeconomic status as indicated by education and financial hardship. The prevalence of current cigarette smokers was highest in black women and lowest in Chinese women. Black women also reported higher exposure to secondhand smoking than women of other race/ethnicities. Chinese and Japanese women consumed seafood and rice more frequently than white or black women.

\section{Analysis of Individual Metals}

The distributions of all 21 metal concentrations ( $\mu \mathrm{g} / \mathrm{L}$ urine and $\mu \mathrm{g} / \mathrm{g}$ creatinine), LODs and detection rates are summarized in Table S1. The percentage of women with detectable concentrations of an individual metal ranged from 2.6 to $100 \%$. Six metals had detection rates less than $70 \%$ (beryllium: $16.2 \%$, chromium: $24.3 \%$, platinum: $2.6 \%$, uranium: $33.0 \%$, vanadium: $37.2 \%$, tungsten: $29.6 \%$ ). The median number of metals detected in SWAN 
participants was 16 . Figure 1 shows the Spearman correlation matrix of the 15 creatinineadjusted metal concentrations for which detection rates were greater or equal than $70 \%$. In general, most metals were modestly and positively correlated with each other. Comparisons of creatinine-adjusted median concentrations of urinary barium, cadmium, cobalt, cesium, mercury, molybdenum, lead, antimony, and thallium in white and black women from SWAN and NHANES 1999-2000 within the same age range are displayed in Figure 2.

The LSGM concentrations of 15 urinary metals detectable in more than $70 \%$ of the participants are shown in Table S2, stratified by race/ethnicity, education, financial hardship, smoking, and secondhand smoking status. Concentration differences for 12 out of 15 metals were observed between race/ethnicity groups. Both Chinese and Japanese women had higher concentrations of arsenic, cadmium, copper, mercury, molybdenum, lead and thallium than white or black women. The most pronounced differences between Asian and white/black women were for arsenic and cadmium. For example, on average, arsenic concentrations were $95.9 \%$ and $121.2 \%$ higher in Chinese than in white and black women, respectively, while cadmium concentrations were $93.0 \%$ higher in Japanese than in white or black women. The highest LSGM concentrations of cobalt, cesium and nickel were also detected in Chinese participants. Women with higher education had lower concentrations of cadmium, antimony and zinc, but higher concentrations of mercury than women with less education. Current smoking status was positively associated with cadmium and lead and inversely associated with cobalt and nickel concentrations. Higher concentrations of mercury and lead were found also among women who had higher exposure to secondhand smoking. No significant differences were observed between metal concentrations and financial hardship.

The LSGM metal concentrations are presented in Table 2 after further adjustment for seafood and rice intake. Higher seafood intake was significantly associated with higher concentrations of arsenic, mercury, molybdenum and lead. Rice intake was also positively associated with arsenic, cesiuMAnd mercury concentrations. Women who consumed two or more seafood meals per week had 55.4\% higher mean concentrations of total arsenic in urine than did women who reported eating seafood less than 1 time per week. For rice, those who consumed 3.5 or more rice meals per week, on average, had $65.5 \%$ higher concentrations of urinary total arsenic compared with participants in the lowest category of rice intake ( $<1$ time per week). To note, seafood and rice intake were only weakly correlated (Spearman correlation $\rho=0.25$ ) (data not shown). The race/ethnic differences in LSGM concentrations of arsenic, cesium, mercury and molybdenum were attenuated but remained significant $(P S<0.05)$ after further adjusting for seafood and rice intake (Table 2, Table S2). Similar race/ethnic differences in metal exposures were observed when LSGM concentrations were compared between white and Chinese women within the Oakland site, and between white and Japanese women within the Los Angeles site (Table S3).

The LSGM concentrations within white and black women are presented in Table 3, stratified by SWAN study sites. In both racial groups, women in Boston had the highest average concentrations of arsenic, cadmium, cesium, mercury and lead; and those at the Pittsburgh site had the highest concentrations of barium, nickel and thallium. Women in southeast Michigan had the lowest average concentrations of arsenic, cadmium and lead. 
After backward elimination, race/ethnicity was selected as a significant predictor for most metals (Table S4). Higher education level remained as a correlate of higher urinary cadmium and mercury concentrations. Being a former or current smoker was significantly associated with both higher cadmium and lead concentrations. Seafood intake remained as an independent predictor of arsenic, cadmium, cesium, mercury and lead. Rice intake was associated also with elevated arsenic, cesium, copper, mercury, molybdenum and nickel concentrations.

\section{Analysis of Exposure Patterns of Metals}

Two clusters of metal exposures were derived by k-means clustering based on the cubic clustering criterion, Elbow method and average Silhouette method (Figure S2), which were labeled as "high" $(\mathrm{n}=562)$ and "low" $(\mathrm{n}=773)$ for the overall metal exposure patterns. Figure 3 shows the mean of each standardized log-transformed creatinine adjusted metal concentration corresponding to the high and low clusters (geometric means can be found in Table S5). Note that standardized concentrations were comparable within each cluster. No cluster had a particularly high or low concentration of specific metals. Odds ratios in the full logistic regression model are presented in Table S6. After backward elimination, being black was associated with higher odds of being clustered into the "low" overall exposure group, while being Chinese or Japanese, being a current smoker, and being in the highest category of seafood intake and rice intake were significantly $(P S<0.05)$ associated with higher odds of being clustered into the "high" group (Table 4).

Use of specific gravity instead of urinary creatinine in models for urine dilution adjustment did not alter our findings significantly (Table S7).

\section{DISCUSSION}

In this study, we evaluated concentrations of 21 metals in urine samples, identified two overall exposure patterns, and examined demographic, socioeconomic, lifestyle, and dietary factors associated with both individual metals and metals exposure patterns, in a large population-based multi-racial/ethnic, multi-site cohort of midlife women in the U.S. Participants sorted into two clusters, suggesting two distinct overall exposure patterns to mixtures of multiple metals in the general environment. Interestingly, each exposure pattern showed homogeneous distributions of individual metals (standardized concentrations). This similarity could be partly explained by the positive correlations among most of the metals we measured in urine samples (Figure 1). One recent study of profiles of environmental chemical mixture exposure among pregnant women using the same k-means clustering method revealed a similar exposure cluster in which some women were consistently exposed to high concentrations of metal (Kalloo et al., 2018). Understanding the exposure patterns of multiple metals is an important first step before evaluating the association between metal mixtures and health outcomes. Our study suggests that k-means clustering is a useful tool to identify exposure clusters in the population.

We observed significant race/ethnic differences in the urinary concentrations of metals, i.e., higher concentrations of arsenic, cadmium, copper, mercury, molybdenum, lead, and thallium in Asian women. Similarly, findings in the U.S. general population for arsenic, 
cadmium, lead, and mercury suggest that Asians had the highest adjusted geometric mean biomarker levels of these metals in NHANES 2011-2012 (Awata et al., 2017a). However, most previous epidemiological studies have focused primarily on "priority toxic metals" while the racial/ethnic differences in metals such as copper and thallium, as well as the overall exposure patterns, have not been adequately captured. Some of the differences in metal concentrations between race/ethnic groups may be related to diet, such as higher intake of seafood and rice reported by Chinese and Japanese study participants. Regular seafood intake, particularly fish and shellfish, contributes to overexposure to methyl mercury, total arsenic, organic arsenic (arsenobetaine and arsenocholine), and lead (Awata et al., 2017b; Bae et al., 2013; Burger and Gochfeld, 2005; Castro-González and MéndezArmenta, 2008; Falcó et al., 2006; Storelli, 2008). Rice consumption has also gained recent attention as a potential source of arsenic exposure (Awata et al., 2017b; Azizur Rahman et al., 2008; M. A. Davis et al., 2012; Gilbert-Diamond et al., 2011; Melkonian et al., 2013). Intake of seafood and rice is an important exposure pathway for explaining racial/ethnic differences in, at least, arsenic and mercury. Stronger associations of seafood and rice intake with both arsenic and mercury were observed within the two Asian populations compared to other race/ethnic populations in this study (data not shown). The seafood intake assessment in this study was based on FFQ, adapted to include ethnic specific foods. However, other seafood items might not have been captured in the standard FFQ that were often served in the Asian diet, such as seaweed, which might be important determinants of the unequal metal distributions across racial groups (Lee et al., 2012). Different metal contamination levels by different types of rice (i.e., white vs. brown rice) may also explain our findings, if the types of rice were different between high and low consumption groups (Consumer Reports, 2014). However, the FFQ used in the current study did not distinguish specific types of rice. Furthermore, Chinese women had even higher concentrations of cobalt, cesium and nickel than Japanese women. The fact that intake of seafood and rice was not different between Chinese and Japanese suggests that other environmental exposure sources or pathways, may differ across Asian populations or that different levels of unmeasured confounding are present within Asian subgroups. The observed differences between Asian and other race/ethnic groups could be confounded by geographic location as both Chinese and Japanese women were sampled only in California, but not at any of the Midwest or Northeast SWAN sites. One recent study conducted in six U.S. cities reported higher arsenic concentrations for participants in the Los Angeles compared to the other cities (Jones et al., 2018). However, we observed distinct race/ethnic differences in metal exposures when LSGM concentrations were compared between white and Chinese women within the Oakland site, and between white and Japanese women within the Los Angeles site (Table S3).

Additionally, we found that geographic location was an important predictor of metal concentrations. White and black women in Boston had higher concentrations of arsenic, cadmium, cesium, mercury and lead than those at other study sites. A higher seafood intake in Boston could potentially account for the observed high metal concentrations. This was supported by the significantly higher seafood intake among white and black women at Boston site compared with other sites in our study population (data not shown). A possible alternative explanation that should be considered is metal contamination of drinking water 
because of an aging infrastructure. For example, lead in the water supply has been attributed to dilapidated drinking water infrastructures, including lead jointed pipelines, end-of-life polyvinyl chloride pipes and household plumbing in communities with aging infrastructures (Hanna-Attisha et al., 2016; Harvey et al., 2015). Lead exposure could also be higher due to older housing stock with lead-based paint another important exposure source (Aschengrau et al., 1997).

High exposure to barium, nickel and thallium were consistently observed in both white and black women in Pittsburgh. Barium is commonly used in metal alloys, colorant in paints, $\mathrm{x}$ ray contrast medium, and naturally occurs in groundwater (ATSDR, 2007a). Barium concentrations in the drinking water were around 10 times higher in regions of Kentucky, northern Illinois, New Mexico, and Pennsylvania in the U.S. (ATSDR, 2007a). Thus drinking water from groundwater sources might be a common route of high exposure to barium among participants in Pittsburgh. Nickel is used in the manufacturing of electronics, metal alloys and batteries. It is released to the atmosphere by combustion of fuel oil, municipal incineration, and industries involved in nickel refining, steel production, and other nickel alloy production (ATSDR, 2005). Based on the emission data in the EPA 1996 National Toxics Inventory database, Pennsylvania had one of the highest average concentrations of nickel in ambient air among the states in the U.S. (ATSDR, 2005). Thallium is another toxic metal that has been widely used in electronics manufacturing in the U.S. Its exposure occurs primarily from industrial processes such as coal-burning and smelting (Peter and Viraraghavan, 2005). Therefore, higher urinary nickel and thallium concentrations among women at Pittsburgh might be attributed to inhalation of contaminated ambient air.

Our study found that several other characteristics are also important predictors of metal concentrations. The observed decreasing trend in cadmium and antimony with increasing education levels accords with previous findings, indicating the role of socioeconomic status in determination of high exposure to environmental toxicants (Tyrrell et al., 2013). However, higher socioeconomic status is not always associated with lower exposure to toxic metals. For example, higher mercury concentrations have been observed in participants with higher education levels because individuals of higher socioeconomic position tend to have higher regular seafood consumption (Awata et al., 2017a; Buchanan et al., 2015; Mortensen et al., 2014). We also observed significant positive associations of cigarette smoking with cadmium and lead concentrations, again demonstrating that cigarette smoking is one of the main sources of cadmium and lead in the general population (ATSDR, 2012; Hu et al., 1996; Richter et al., 2013). Secondhand smoking also contributed to increased urinary lead concentration, providing support for its role as a modifiable source of lead exposure not only in children and adolescents reported previously (Apostolou et al., 2012), but in midlife women.

In this study, the creatinine-adjusted median concentrations of most metals were comparable to the concentrations in women of the same age range (45-56 years) from NHANES 19992000. Median concentrations of molybdenum in white and black women and of mercury in black women in SWAN women seem to be a little bit higher than those in NHANES. Seafood intake was shown to be a significant source of both mercury and molybdenum in 
this study. Higher seafood consumption, especially in an area like Boston that reported the highest seafood intake in our analysis, might account for the high metal concentrations in SWAN participants compared with those in NHANES. Significantly higher LSGM concentrations of mercury and molybdenum were also observed among women at Boston site compared with other sites in our study (Table 3). However, we cannot rule out other possible exposure pathways that may account for the observed differences. Nonetheless, our understanding of sources of metal exposure remains incomplete, however, these findings prompt follow-up In a future study.

Our study has several limitations. First, the metals we measured in this study have various half-lives in the human body. Urinary concentrations of metals with short half-lives such as arsenic, barium, cobalt, cesium, and thallium mainly reflect recent exposures (ATSDR, 2007a, 2007b, 2004a, 2004b, 1992) and may depend on the participants' food consumption within a few days previous to the urine sample collection (Navas-Acien et al., 2011). In contrast, other metals such as cadmium are not rapidly excreted and have very long halflives from years to decades (ATSDR, 2012). As health endpoints related to metals are likely affected by exposures over time-periods longer than a few days, information on the temporal variability of urinary metals concentrations, especially for those with short half-lives, is needed to characterize average metals exposures over time in epidemiological studies. Second, urine may not be an optimal biological matrix for some metals, such as lead. However, urinary lead adjusted for creatinine has been suggested as a good proxy for plasma lead, where plasma lead is the most toxicologically active lead component but is difficult to measure accurately due to the extremely low concentrations and possible contamination from various sources (Tsaih et al., 2001, 1999). Third, in our study only total arsenic concentrations were measured; arsenic speciation data were not available. The source and toxicity of different arsenic species vary. Major sources of inorganic arsenic in the general population are contaminated drinking water and rice intake (Gilbert-Diamond et al., 2011; Hughes et al., 2011). Inorganic arsenic has been associated with adverse health outcomes such as cardiovascular disease, diabetes, and some cancers (Chen et al., 2013; Maull et al., 2012; Meliker et al., 2010; Steinmaus et al., 2014). Seafood intake is a major source of organic arsenic (Jones et al., 2016), which is generally considered to have low toxicity (Cullen and Reimer, 1989). Arsenic speciation would improve assessment of arsenic exposures and associated health risks. Fourth, seafood and rice intake in this study was obtained from an FFQ adm inistered at the SWAN baseline (1996-1997), whereas the urine samples were collected at SWAN visit 03 (1999-2000). This FFQ would not capture possible dietary changes that may have occured during the 3-year gap before urine sample collection although rapid diet changes are not very likely in this age group (Weismayer et al., 2006). Dietary assessments were self-reported, and thus subject to recall bias. However, statistical adjustment for self-reported energy intake in our regression models helped to reduce the influence of response biases since measurement error in both energy intake and food intake estimates are correlated (Subar et al., 2015). Finally, participants in our study were midlife women. Thus the present findings may not be generalizable to $\mathrm{m}$ en or women at different lifestages.

This study also has numerous strengths. We systematically examined a suite of 21 metals in urine samples in midlife women for whom scant exposure data is available. We used a data- 
driven clustering approach to summarizing information of multiple environmental exposures into distinct metal clusters. This approach proved to be a useful tool as it identified different overall exposure patterns of metals and the underlying grouping of metals that may be useful for future evaluations of metal-mixtures health effects. Further, the wide geographical and racial/ethnic coverage of the SWAN participants enabled us to compare differences in biomarkers levels across multiple groups and increased the generalizability of our findings.

In conclusion, we observed marked differences in distributions of a comprehensive set of metals and in the overall metal exposure patterns, by race/ethnicity, education, smoking, secondhand smoking, seafood intake, rice intake and geographic sites, among midlife women from the U.S. general population, as represented by participants of SWAN. Chinese and Japanese women, had higher urinary concentrations of arsenic, cadmium, copper, mercury, molybdenum, lead, thallium, compared with other race/ethnic groups. Women in the "high" overall exposure pattern were more likely to be Asians and less likely to be black. We confirmed that seafood intake and rice intake were important dietary sources of toxic metals including arsenic, lead, cadmium and mercury, which could also explain the observed racial differences in arsenic and mercury. Education, smoking, secondhand smoking and geographic sites were significant predictors of urinary concentrations of different sets of metals. Additional studies are needed to examine other potential sources and characterizations of metal exposures, to better understand racial/ethnic inequalities in environmental metal exposures. Further research is also needed to investigate whether the observed race/ethnic differences in metal exposures may contribute to differences in health outcomes.

\title{
Supplementary Material
}

Refer to Web version on PubMed Central for supplementary material.

\section{Acknowledgements}

This study was supported by grants from the National Institute of Environmental Health Sciences (NIEHS) R01ES026578, R01-ES026964 and P30-ES017885, and by the Center for Disease Control and Prevention (CDC)/ National Institute for Occupational Safety and Health (NIOSH) grant T42-OH008455.

\begin{abstract}
The Study of Women's Health Across the Nation (SWAN) has grant support from the National Institutes of Health (NIH), DHHS, through the National Institute on Aging (NIA), the National Institute of Nursing Research (NINR) and the NIH Office of Research on Women's Health (ORWH) (Grants U01NR004061; U01AG012505, U01AG012535, U01AG012531, U01AG012539, U01AG012546, U01AG012553, U01AG012554, U01AG012495). The ontent of this article is solely the responsibility of the authors and does not necessarily represent the official views of the NIA, NINR, ORW H or the NIH. The SWAN Repository (U01AG017719). This publication was supported in part by the National Center for Research Resources and the National Center for Advancing Translational Sciences, National Institutes of Health, through UCSF-CTSI Grant Number UL1 RR024131.

Clinical Centers: University of Michigan, Ann Arbor - Siobán Harlow, PI 2011 - present, MaryFran Sowers, PI 1994-2011; Massachusetts General Hospital, Boston, MA - Joel Finkelstein, PI 1999 - present; Robert Neer, PI 1994 - 1999; Rush University, Rush University Medical Center, Chicago, IL - Howard Kravitz, PI 2009 - present; Lynda Powell, PI 1994 - 2009; University of California, Davis/Kaiser - Ellen Gold, PI; University of California, Los Angeles - Gail Greendale, PI; Albert Einstein College of Medicine, Bronx, NY - Carol Derby, PI 2011 present, Rachel Wildman, PI 2010 - 2011; Nanette Santoro, PI 2004 - 2010; University Of Medicine and Dentistry - New Jersey Medical School, Newark - Gerson Weiss, PI 1994 - 2004; and the University of Pittsburgh, Pittsburgh, $P A$ - Karen Matthews, PI.
\end{abstract}


NIH Program Office: National Institute on Aging, Bethesda, MD - Chhanda Dutta 2016- present; Winifred Rossi 2012-2016; Sherry Sherman 1994 - 2012; Marcia Ory 1994 - 2001; National Institute of Nursing Research, Bethesda, MD - Program Officers.

Central Laboratory: University of Michigan, Ann Arbor - Daniel McConnell (Central Ligand Assay Satellite Services).

SWAN Repository: University of Michigan, Ann Arbor - Siobán Harlow 2013 - Present; Dan McConnell 2011 2013; MaryFran Sowers 2000 - 2011.

Coordinating Center: University of Pittsburgh, Pittsburgh, PA - Maria Mori Brooks, PI 2012 - present; Kim SuttonTyrrell, PI 2001 - 2012; New England Research Institutes, Watertown, MA - Sonja McKinlay, PI 1995 - 2001.

Steering Committee: Susan Johnson, Current Chair

Chris Gallagher, Former Chair

We thank the study staff at each site and all the women who participated in SWAN.

\section{REFERENCES}

Alloway BJ, 2013 Sources of Heavy Metals and Metalloids in Soils. Springer, Dordrecht, pp. 11-50. 10.1007/978-94-007-4470-7_2

Apostolou A, Garcia-Esquinas E, Fadrowski JJ, McLain P, Weaver VM, Navas-Acien A, Navas-Acien A, 2012 Secondhand tobacco smoke: a source of lead exposure in US children and adolescents. Am. J. Public Health 102, 714-22. 10.2105/AJPH.2011.300161 [PubMed: 21852639]

Aschengrau A, Beiser A, Bellinger D, Copenhafer D, Weitzman M, 1997 Residential lead-based-paint hazard remediation and soil lead abatement: their impact among children with mildly elevated blood lead levels. Am. J. Public Health 87, 1698-702. 10.2105/AJPH.87.10.1698 [PubMed: 9357358]

ATSDR, 2012 Toxicological profile for Cadmium. [WWW Document]. URL https:// www.atsdr.cdc.gov/toxprofiles/tp.asp?id=48\&tid=15 (accessed 4.23.18).

ATSDR, 2007a Toxicological profile for Barium. [WWW Document]. URL https://www.atsdr.cdc.gov/ toxprofiles/tp.asp?id=327\&tid=57 (accessed 4.27.18).

ATSDR, 2007b Toxicological profile for Lead. [WWW Document]. URL https://www.atsdr.cdc.gov/ toxprofiles/tp.asp?id=96\&tid=22 (accessed 4.27.18).

ATSDR, 2005 Toxicological profile for Nickel. [WWW Document]. URL https://www.atsdr.cdc.gov/ toxprofiles/TP.asp?id=245\&tid=44 (accessed 4.19.18).

ATSDR, 2004a Toxicological profile for Cesium [WWW Document]. URL https://www.atsdr.cdc.gov/ toxprofiles/TP.asp?id=578\&tid=107 (accessed 4.27.18).

ATSDR, 2004b Toxicological profile for cobalt. [WWW Document]. URL https://www.atsdr.cdc.gov/ toxprofiles/tp.asp?id=373\&tid=64 (accessed 4.27.18).

ATSDR, 1992 Toxicological profile for Thallium. [WWW Document]. URL https:// www.atsdr.cdc.gov/toxprofiles/tp.asp?id=309\&tid=49 (accessed 4.27.18).

Awata H, Linder S, Mitchell LE, Delclos GL, 2017a Biomarker Levels of Toxic Metals among Asian Populations in the United States: NHANES 2011-2012. Environ. Health Perspect 125, 306-313. 10.1289/EHP27 [PubMed: 27517362]

Awata H, Linder S, Mitchell LE, Delclos GL, 2017b Association of Dietary Intake and Biomarker Levels of Arsenic, Cadmium, Lead, and Mercury among Asian Populations in the United States: NHANES 2011-2012. Environ. Health Perspect. 125, 314-323. 10.1289/EHP28 [PubMed: 27586241]

Azizur Rahman M, Hasegawa H, Mahfuzur Rahman M, Mazid Miah MA, Tasmin A,2008 Arsenic accumulation in rice (Oryza sativa L.): Human exposure through food chain. Ecotoxicol. Environ. Saf 69, 317-324. 10.1016/J.EC0ENV.2007.01.005 [PubMed: 17346792]

Bae H-S, Ryu D-Y, Choi B-S, Park J-D, 2013 Urinary Arsenic Concentrations and their Associated Factors in Korean Adults. Toxicol. Res 29, 137-142. 10.5487/TR.2013.29.2.137 [PubMed: 24278640] 
Barr DB, Wilder LC, Caudill SP, Gonzalez AJ, Needham LL, Pirkle JL, 2005 Urinary creatinine concentrations in the U.S. population: implications for urinary biologic monitoring measurements. Environ. Health Perspect 113, 192-200. 10.1289/ehp.7337 [PubMed: 15687057]

Birnbaum LS, 2018 Moving NIEHS Forward for the Next Five Years. Environ. Health Perspect. 126, 091001 10.1289/EHP4356

Block G, Hartman AM, Dresser CM, Carroll MD, Gannon J, Gardner L, 1986 A data- based approach to diet questionnaire design and testing. Am. J. Epidemiol 124, 453-69. [PubMed: 3740045]

Bosch AC, O’Neill B, Sigge GO, Kerwath SE, Hoffman LC, 2016 Heavy metals in marine fish meat and consumer health: a review. J. Sci. Food Agric. 96, 32-48. 10.1002/jsfa.7360 [PubMed: 26238481]

Buchanan S, Anglen J, Turyk M, 2015 Methyl mercury exposure in populations at risk: Analysis of NHANES 2011-2012. Environ. Res 140, 56-64. 10.1016/j.envres.2015.03.005 [PubMed: 25825131]

Burger J, Gochfeld M, 2005 Heavy metals in commercial fish in New Jersey. Environ. Res 99, 403412. 10.1016/J.ENVRES.2005.02.001 [PubMed: 16307983]

Calafat AM, 2012 The U.S. National Health and Nutrition Examination Survey and human exposure to environmental chemicals. Int. J. Hyg. Environ. Health 215, 99-101. 10.1016/j.ijheh.2011.08.014 [PubMed: 21937270]

Castro-González MI, Méndez-Armenta M, 2008 Heavy metals: Implications associated to fish consumption. Environ. Toxicol. Pharmacol 26, 263-271. 10.1016/J.ETAP.2008.06.001 [PubMed: 21791373]

CDC/NCHS, 2018 NHANES - Questionnaires, Datasets, and Related Documentation [WWW Document]. URL https://wwwn.cdc.gov/nchs/nhanes/Default.aspx (accessed 6.14.18).

CDC, 2012 Laboratory Procedure Manual, Multi-Element in urine. NHANES 2011-2012.

Chen Y, Wu F, Liu M, Parvez F, Slavkovich V, Eunus M, Ahmed A, Argos M, Islam T, RakibuzZaman M, Hasan R, Sarwar G, Levy D, Graziano J, Ahsan H, 2013 A Prospective Study of Arsenic Exposure, Arsenic Methylation Capacity, and Risk of Cardiovascular Disease in Bangladesh. Environ. Health Perspect 121, 832-838. 10.1289/ehp.1205797 [PubMed: 23665672]

Consumer Reports, 2014 How much arsenic is in your rice? [WWW Document]. URL https:// www.consumerreports.org/cro/magazine/2015/01/how-much-arsenic-is-in-your-rice/index.htm (accessed 1.16.19).

Cullen WR, Reimer KJ, 1989 Arsenic speciation in the environment. Chem. Rev 89, 713764. 10.1021/ cr00094a002

Davis MA, Mackenzie TA, Cottingham KL, Gilbert-Diamond D, Punshon T, Karagas MR, 2012 Rice consumption and urinary arsenic concentrations in U.S. children. Environ. Health Perspect 120, 1418-24. 10.1289/ehp.1205014 [PubMed: 23008276]

Davis SR, Castelo-Branco C, Chedraui P, Lumsden MA, Nappi RE, Shah D, Villaseca P, Writing Group of the International Menopause Society for World Menopause Day 2012, 2012 Understanding weight gain at menopause. Climacteric 15, 419-429. 10.3109/13697137.2012.707385 [PubMed: 22978257]

Ding N, Wang X, Weisskopf MG, Sparrow D, Schwartz J, Hu H, Park SK, 2016 Lead-Related Genetic Loci, cumulative lead exposure and incident coronary heart disease: The normative aging study. PLoS One 11, 1-18. 10.1371/journal.pone.0161472

Falcó G, Llobet JM, Bocio A, Domingo JL, 2006 Daily Intake of Arsenic, Cadmium, Mercury, and Lead by Consumption of Edible Marine Species. J. Agric. Food Chem 54, 6106-6112. 10.1021/ jf0610110 [PubMed: 16881724]

Fraga CG, 2005 Relevance, essentiality and toxicity of trace elements in human health. Mol. Aspects Med 26, 235-244. 10.1016/j.mam.2005.07.013 [PubMed: 16125765]

Gilbert-Diamond D, Cottingham KL, Gruber JF, Punshon T, Sayarath V, Gandolfi AJ, Baker ER, Jackson BP, Folt CL, Karagas MR, 2011 Rice consumption contributes to arsenic exposure in US women. Proc. Natl. Acad. Sci. U. S. A 108, 20656-60. 10.1073/pnas.1109127108 [PubMed: 22143778] 
Hall M, Buysse DJ, Nofzinger EA, Reynolds CF, Thompson W, Mazumdar S, Monk TH, 2008 Financial strain is a significant correlate of sleep continuity disturbances in late-life. Biol. Psychol 77, 217-222. 10.1016/J.BIOPSYCH0.2007.10.012 [PubMed: 18055094]

Hanna-Attisha M, LaChance J, Sadler RC, Champney Schnepp A, 2016 Elevated Blood Lead Levels in Children Associated With the Flint Drinking Water Crisis: A Spatial Analysis of Risk and Public Health Response. Am. J. Public Health 106, 283-90. 10.2105/AJPH.2015.303003 [PubMed: 26691115]

Harvey PJ, Handley HK, Taylor MP, 2015 Identification of the sources of metal (lead) contamination in drinking waters in north-eastern Tasmania using lead isotopic compositions. Environ. Sci. Pollut. Res 22, 12276-12288. 10.1007/s11356-015-4349-2

Hernandez-Avila M, Villalpando CG, Palazuelos E, Hu H, Villalpando ME, Martinez DR, 2000 Determinants of blood lead levels across the menopausal transition. Arch. Environ. Health 55, 355-60. 10.1080/00039890009604028 [PubMed: 11063411]

Hu H, Payton M, Korrick S, Aro A, Sparrow D, Weiss ST, Rotnitzky A, 1996 Determinants of bone and blood lead levels among community-exposed middle-aged to elderly men. The normative aging study. Am. J. Epidemiol 144, 749-59. [PubMed: 8857824]

Hughes MF, Beck BD, Chen Y, Lewis AS, Thomas DJ, 2011 Arsenic Exposure and Toxicology: A Historical Perspective. Toxicol. Sci 123, 305-332. 10.1093/toxsci/kfr184 [PubMed: 21750349]

Jain AK, 2010 Data clustering: 50 years beyond K-means. Pattern Recognit. Lett 31, 651-666. 10.1016/J.PATREC.2009.09.011

Jones MR, Tellez-Plaza M, Vaidya D, Grau-Perez M, Post WS, Kaufman JD, Guallar E, Francesconi KA, Goessler W, Nachman KE, Sanchez TR, Navas-Acien A, 2018 Ethnic, geographic and dietary differences in arsenic exposure in the multi-ethnic study of atherosclerosis (MESA). J. Expo. Sci. Environ. Epidemiol 10.1038/s41370-018-0042-0

Jones MR, Tellez-Plaza M, Vaidya D, Grau M, Francesconi KA, Goessler W, Guallar E, Post WS, Kaufman JD, Navas-Acien A, 2016 Estimation of Inorganic Arsenic Exposure in Populations With Frequent Seafood Intake: Evidence From MESA and NHANES. Am. J. Epidemiol 184, 590-602. 10.1093/aje/kww097 [PubMed: 27702745]

Kalloo G, Wellenius GA, McCandless L, Calafat AM, Sjodin A, Karagas M, Chen A, Yolton K, Lanphear BP, Braun JM, 2018 Profiles and Predictors of Environmental Chemical Mixture Exposure among Pregnant Women: The Health Outcomes and Measures of the Environment Study. Environ. Sci. Technol 52, 10104-10113. 10.1021/acs.est.8b02946 [PubMed: 30088764]

Kim C, 2012 Does menopause increase diabetes risk? Strategies for diabetes prevention in midlife women. Womens. Health (Lond. Engl). 8, 155-67. 10.2217/whe.11.95 [PubMed: 22375719]

Korrick SA, Hunter DJ, Rotnitzky A, Hu H, Speizer FE, 1999 Lead and hypertension in a sample of middle-aged women. Am. J. Public Health 89, 330-5. 10.2105/AJPH.89.3.330 [PubMed: 10076481]

Lanphear BP, Rauch S, Auinger P, Allen RW, Hornung RW, 2018 Low-level lead exposure and mortality in US adults: a population-based cohort study. Lancet. Public Heal. 3, e177-e184. 10.1016/S2468-2667(18)30025-2

Lee JW, Lee CK, Moon CS, Choi IJ, Lee KJ, Yi S-M, Jang B-K, Yoon B. jun, Kim DS, Peak D, Sul D, Oh E, Im H, Kang HS, Kim J, Lee J-T, Kim K, Park KL, Ahn R, Park SH, Kim SC, Park C-H, Lee JH, 2012. Korea National Survey for Environmental Pollutants in the Human Body 2008: Heavy metals in the blood or urine of the Korean population. Int. J. Hyg. Environ. Health 215, 449-457. 10.1016/J.IJHEH.2012.01.002 [PubMed: 22341685]

Maull EA, Ahsan H, Edwards J, Longnecker MP, Navas-Acien A, Pi J, Silbergeld EK, Styblo M, Tseng C-H, Thayer KA, Loomis D, 2012 Evaluation of the Association between Arsenic and Diabetes: A National Toxicology Program W orkshop Review.Environ. Health Perspect. 120, 1658-1670. 10.1289/ehp.1104579 [PubMed: 22889723]

Meliker JR, Slotnick MJ, AvRuskin GA, Schottenfeld D, Jacquez GM, Wilson ML, Goovaerts P, Franzblau A, Nriagu JO, 2010 Lifetime exposure to arsenic in drinking water and bladder cancer: a population-based case-control study in Michigan, USA. Cancer Causes Control 21, 745-757. 10.1007/s10552-010-9503-z [PubMed: 20084543] 
Melkonian S, Argos M, Hall MN, Chen Y, Parvez F, Pierce B, Cao H, Aschebrook-Kilfoy B, Ahmed A, Islam T, Slavcovich V, Gamble M, Haris PI, Graziano JH, Ahsan H, 2013 Urinary and Dietary Analysis of 18,470 Bangladeshis Reveal a Correlation of Rice Consumption with Arsenic Exposure and Toxicity. PLoS One 8, e80691 10.1371/journal.pone.0080691 [PubMed: 24260455]

Mohammed Abdul KS, Jayasinghe SS, Chandana EPS, Jayasumana C, De Silva PMCS, 2015. Arsenic and human health effects: A review. Environ. Toxicol. Pharmacol 40, 828-846. 10.1016/j.etap. 2015.09.016 [PubMed: 26476885]

Mohod CV, Dhote J, 2013 REVIEW OF HEAVY METALS IN DRINKING WATER AND THEIR EFFECT ON HUMAN HEALTH. Int. J. Innov. Res. Sci. , Eng. Technol 2.

Mortensen ME, Caudill SP, Caldwell KL, Ward CD, Jones RL, 2014. Total and methyl mercury in whole blood measured for the first time in the U.S. population: NHANES 2011-2012. Environ. Res 134, 257-64. 10.1016/j.envres.2014.07.019 [PubMed: 25173092]

Navas-Acien A, Francesconi KA, Silbergeld EK, Guallar E, 2011. Seafood intake and urine concentrations of total arsenic, dimethylarsinate and arsenobetaine in the US population. Environ. Res 111, 110-8. 10.1016/j.envres.2010.10.009 [PubMed: 21093857]

Navas-Acien A, Guallar E, Silbergeld EK, Rothenberg SJ, 2007 Lead Exposure and Cardiovascular Disease: A Systematic Review. Environ. Health Perspect. 115, 472-482. [PubMed: 17431501]

O'Brien KM, Upson K, Cook NR, Weinberg CR, 2016 Environmental Chemicals in Urine and Blood: Improving Methods for Creatinine and Lipid Adjustment. Environ. Health Perspect. 124, 220-7. 10.1289/ehp.1509693 [PubMed: 26219104]

Pang Y, Peng RD, Jones MR, Francesconi KA, Goessler W, Howard BV, Umans JG, Best LG, Guallar E, Post WS, Kaufman JD, Vaidya D, Navas-Acien A, 2016. Metal mixtures in urban and rural populations in the US: The Multi-Ethnic Study of Atherosclerosis and the Strong Heart Study. Environ. Res 147, 356-364. 10.1016/j.envres.2016.02.032 [PubMed: 26945432]

Park SK, Tao Y, Meeker JD, Harlow SD, Mukherjee B, 2014 Environmental Risk Score as a New Tool to Examine Multi-Pollutants in Epidemiologic Research: An Example from the NHANES Study Using Serum Lipid Levels. PLoS One 9, e98632 10.1371/journal.pone.0098632 [PubMed: 24901996]

Park SK, Zhao Z, Mukherjee B, 2017 Construction of environmental risk score beyond standard linear models using machine learning methods: application to metal mixtures, oxidative stress and cardiovascular disease in NHANES. Environ. Heal 16, 102 10.1186/s12940-017-0310-9

Peter ALJ, Viraraghavan T, 2005 Thallium: a review of public health and environmental concerns. Environ. Int 31, 493-501. 10.1016/J.ENVINT.2004.09.003 [PubMed: 15788190]

Polotsky H, Polotsky A, 2010 Metabolic Implications of Menopause. Semin. Reprod. Med 28, 426434. 10.1055/s-0030-1262902 [PubMed: 20865657]

Richter PA, Bishop EE, Wang J, Kaufmann R, 2013 Trends in tobacco smoke exposure and blood lead levels among youths and adults in the United States: the National Health and Nutrition Examination Survey, 1999-2008. Prev. Chronic Dis. 10, E213 10.5888/pcd10.130056 [PubMed: 24355106]

Rousseeuw PJ, 1987 Silhouett.es: A graphical aid to the interpretation and validation of cluster analysis. J. Comput. Appl. Math 20, 53-65. 10.1016/0377-0427(87)90125-7

Santoro N, Sutton-Tyrrell K, Sutton-Tyrrell K, 2011 The SWAN song: Study of Women's Health Across the Nation's recurring themes. Obstet. Gynecol. Clin. North Am. 38, 417-23. 10.1016/ j.ogc.2011.05.001 [PubMed: 21961710]

Satarug S, Garrett SH, Sens MA, Sens DA, 2009 Cadmium, Environmental Exposure, and Health Outcomes. Environ. Health Perspect. 118, 182-190. 10.1289/ehp.0901234

Sowers MF, Crawford SL, Sternfeld B, Morganstein D, Gold EB, Greendale GA, Evans D, Neer R, Matthews K, Sherman S, Lo A, Weiss G, Kelsey J, 2000 SWAN: a multi-center, multi-ethnic, community-based cohort study of women and the menopausal transition, in: Lobo RA, Kelsey J, Marcus R (Eds.), Menopause: Biology and Pathobiology. Academic Press, pp. 175-188.

Steinmaus C, Ferreccio C, Yuan Y, Acevedo J, González F, Perez L, Cortés S, Balmes JR, Liaw J, Smith AH, 2014 Elevated Lung Cancer in Younger Adults and Low Concentrations of Arsenic in Water. Am. J. Epidemiol 180, 1082-1087. 10.1093/aje/kwu238 [PubMed: 25371173] 
Storelli MM, 2008 Potential human health risks from metals $(\mathrm{Hg}, \mathrm{Cd}$, and $\mathrm{Pb}$ ) and polychlorinated biphenyls (PCBs) via seafood consumption: Estimation of target hazard quotients (THQs) and toxic equivalents (TEQs). Food Chem. Toxicol 46, 2782-2788. 10.1016/J.FCT.2008.05.011 [PubMed: 18584931]

Stuenkel CA, 2017. Menopause, hormone therapy and diabetes. Climacteric 20, 11-21. 10.1080/13697137.2016.1267723 [PubMed: 28064520]

Subar AF, Freedman LS, Tooze JA, Kirkpatrick SI, Boushey C, Neuhouser ML, Thompson FE, Potischman N, Guenther PM, Tarasuk V, Reedy J, Krebs-Smith SM, 2015 Addressing Current Criticism Regarding the Value of Self-Report Dietary Data. J. Nutr 145, 2639-2645. 10.3945/jn. 115.219634 [PubMed: 26468491]

Theppeang K, Glass TA, Bandeen-Roche K, Todd AC, Rohde CA, Schwartz BS, 2008 Gender and race/ethnicity differences in lead dose biomarkers. Am. J. Public Health 98, 1248-55. 10.2105/ AJPH.2007.118505 [PubMed: 18511728]

Tsaih SW, Korrick S, Schwartz J, Lee ML, Amarasiriwardena C, Aro A, Sparrow D, Hu H, 2001 Influence of bone resorption on the mobilization of lead from bone among middle-aged and elderly men: the Normative Aging Study. Environ. Health Perspect. 109, 995-9. [PubMed: 11675263]

Tsaih SW, Schwartz J, Lee ML, Amarasiriwardena C, Aro A, Sparrow D, Hu H, 1999 The independent contribution of bone and erythrocyte lead to urinary lead among middle-aged and elderly men: the normative aging study. Environ. Health Perspect. 107, 391-6.

Tyrrell J, Melzer D, Henley W, Galloway TS, Osborne NJ, 2013 Associations between socioeconomic status and environmental toxicant concentrations in adults in the USA: NHANES 2001-2010. Environ. Int 59, 328-335. 10.1016/j.envint.2013.06.017 [PubMed: 23892225]

Wang X, Ding N, Tucker KL, Weisskopf MG, Sparrow D, Hu H, Park SK, 2017 A Western Diet Pattern Is Associated with Higher Concentrations of Blood and Bone Lead among Middle-Aged and Elderly Men. J. Nutr. jn 249060 10.3945/jn.117.249060

Wang X, Mukherjee B, Park SK, 2018 Associations of cumulative exposure to heavy metal mixtures with obesity and its comorbidities among U.S. adults in NHANES 2003-2014. Environ. Int 121, 683-694. 10.1016/J.ENVINT.2018.09.035 [PubMed: 30316184]

Warren S, Sarie S, 1983 Cubic clustering criterion.

Weismayer C, Anderson JG, Wolk A, 2006 Changes in the Stability of Dietary Patterns in a Study of Middle-Aged Swedish Women. J. Nutr 136, 1582-1587. 10.1093/jn/136.6.1582 [PubMed: 16702325]

Willett WC, Howe GR, Kushi LH, 1997 Adjustment for total energy intake in epidemiologic studies. Am. J. Clin. Nutr 65, 1220S-1228S. 10.1093/ajcn/65.4.1220S [PubMed: 9094926]

Zahir F, Rizwi SJ, Haq SK, Khan RH, 2005 Low dose mercury toxicity and human health. Environ. Toxicol. Pharmacol 20, 351-360. 10.1016/J.ETAP.2005.03.007 [PubMed: 21783611] 


\section{Highlights}

- Distributions and determinants of 21 urinary metal concentrations were examined in midlife women.

- Two distinct overall metal exposure patterns- "high" vs. "low" were identified.

- Metal distributions differ by racial/ethnic, sociodemographic, lifestyle, and geographic characteristics.

- Asian women experienced the highest exposures to multiple metals compared with other racial/ethnic groups. 


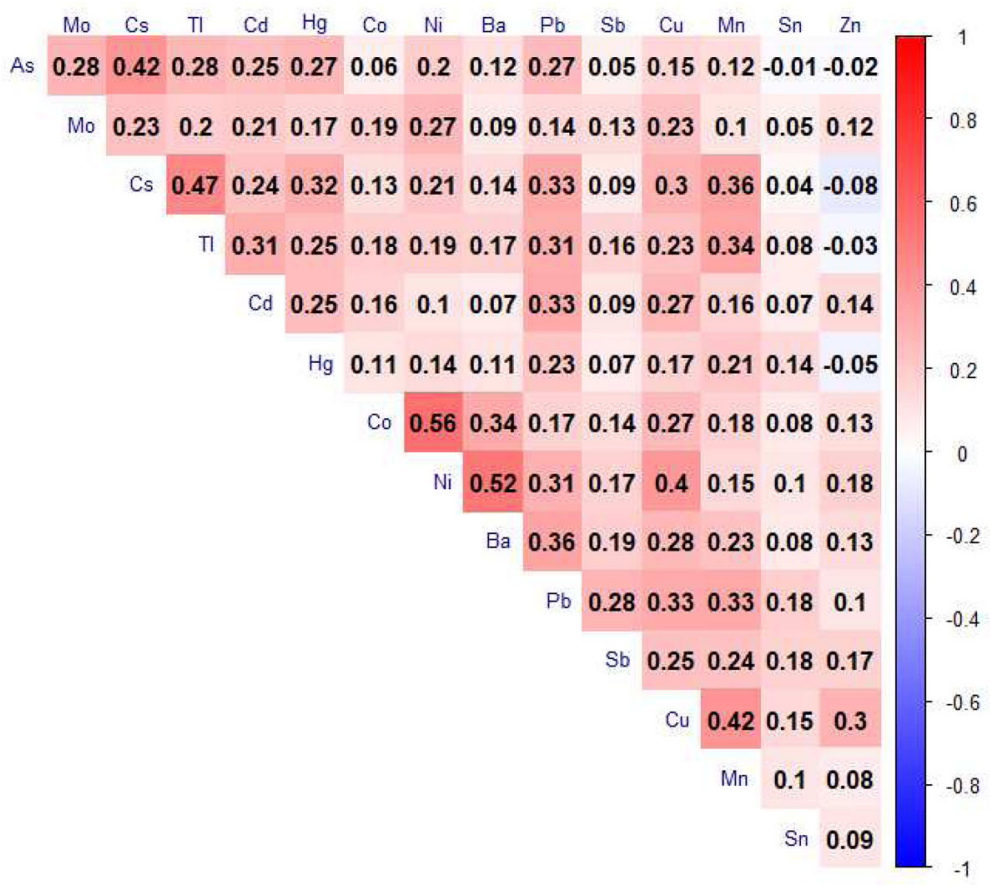

Figure 1.

Spearman correlation matrix of urinary creatinine-adjusted metal concentrations. As: arsenic, Ba: barium, Cd: cadmium, Co: cobalt, Cs: cesium, $\mathrm{Cu}$ : copper, $\mathrm{Hg}$ : mercury, $\mathrm{Mn}$ : manganese, Mo: molybdenum, Ni: nickel, Pb: lead, Sb: antimony, Sn: tin, Tl: thallium, Zn: zinc. 




Figure 2.

Comparisons of creatinine-adjusted median concentrations of urinary metals in white and black women from SWAN and NHANES 1999-2000. NHANES: National Health and Nutrition Examination Survey. Ba: barium, Cd: cadmium, Co: cobalt, Cs: cesium, Hg: mercury, Mo: molybdenum, $\mathrm{Pb}$ : lead, $\mathrm{Sb}$ : antimony, Tl: thallium. 
A.

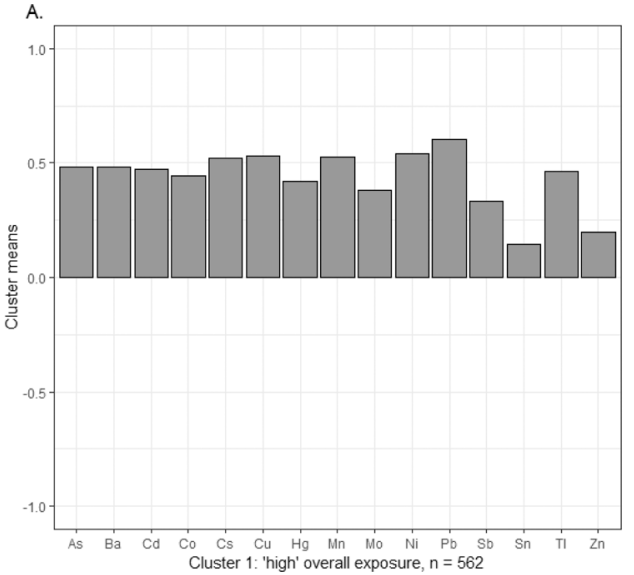



Figure 3.

Cluster means of the 15 standardized log-transformed urinary metals using k-means in the SWAN data. Y-axis (cluster means) represents the mean standardized natural logtransformed urinary creatinine adjusted metal concentrations. Cluster 1: "high" overall metal exposure pattern; cluster 2: "low" overall metal exposure pattern. As: arsenic, Ba: barium, $\mathrm{Cd}$ : cadmium, Co: cobalt, Cs: cesium, $\mathrm{Cu}$ : copper, $\mathrm{Hg}$ : mercury, Mn: manganese, Mo: molybdenum, Ni: nickel, $\mathrm{Pb}$ : lead, Sb: antimony, $\mathrm{Sn}$ : tin, Tl: thallium, $\mathrm{Zn}$ : zinc. 


\section{옴 \\ }



Int J Hyg Environ Health. Author manuscript; available in PMC 2020 June 01. 







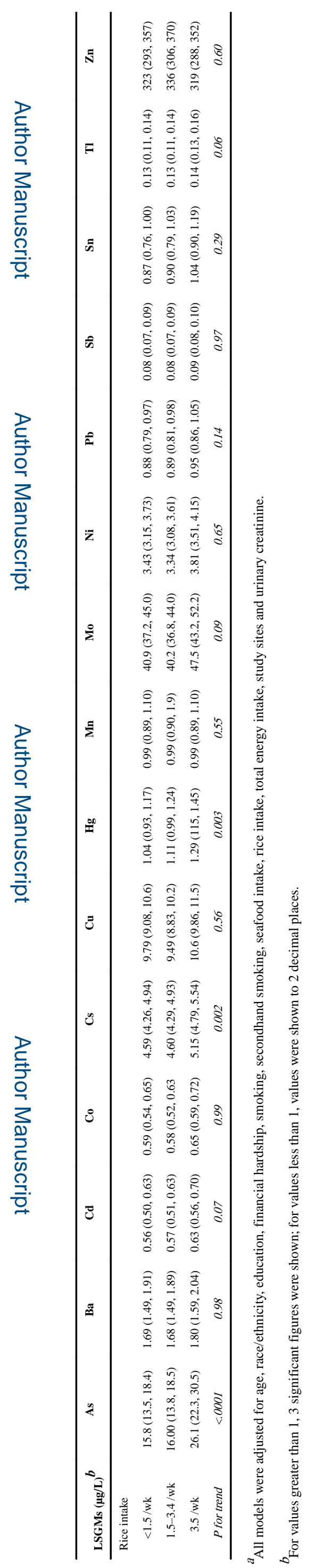




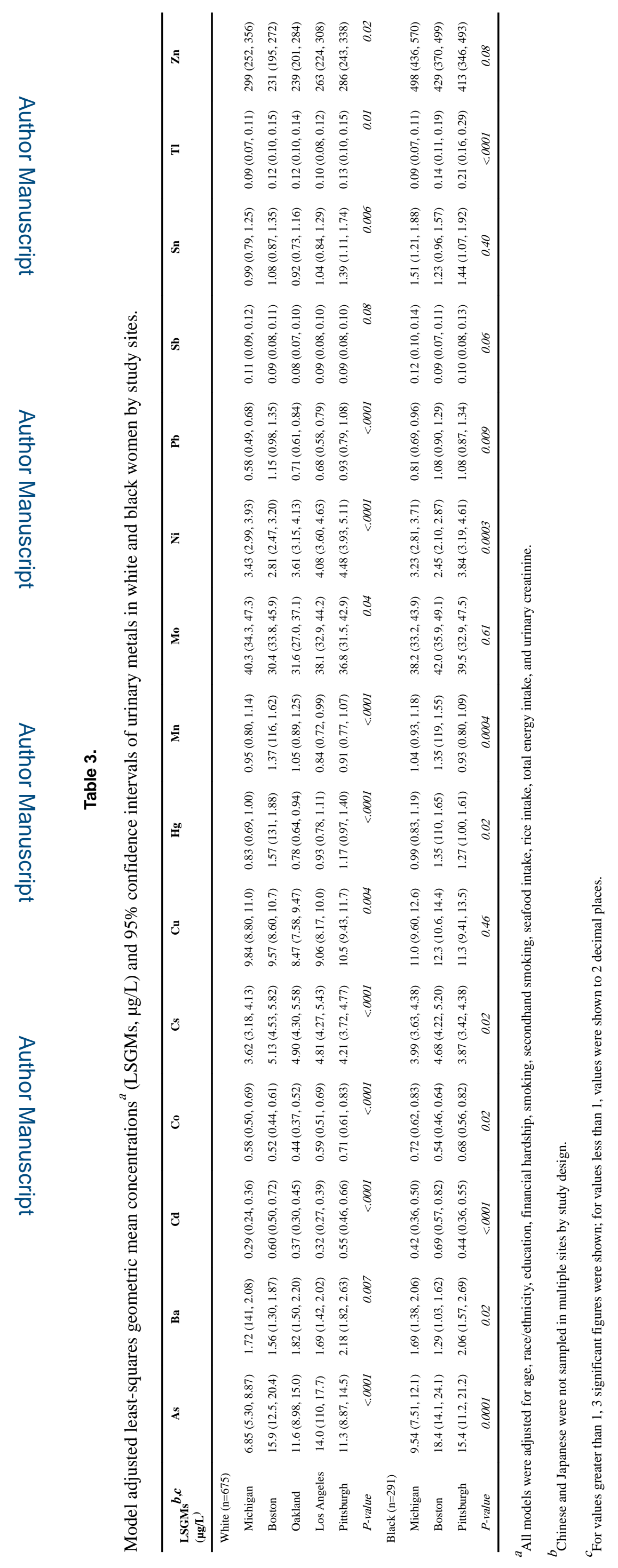


Table 4.

Estimated cumulative odds ratio (95\% confidence intervals) of being clustered into the "high" exposure pattern $^{a}$ by selected determinants in backward elimination ${ }^{b}$.

\begin{tabular}{lccc}
\hline Selected variables & Odds ratio & 95\% CI & P-value $^{\boldsymbol{d}}$ \\
\hline Black vs. white women & 0.39 & $0.26,0.56$ & $<.0001$ \\
Chinese vs. white women & 2.10 & $1.19,3.69$ & 0.01 \\
Japanese vs. white women & 2.32 & $1.39,3.90$ & 0.001 \\
Former vs. never smoker & 1.03 & $0.78,1.37$ & 0.84 \\
Current vs. never smoker & 2.25 & $1.47,3.44$ & 0.0002 \\
Seafood intake $1-1.9 /$ wk vs. $<1$ time/wk & 1.31 & $0.96,1.77$ & 0.09 \\
Seafood intake $\geq 2$ vs. $<1$ time/wk & 1.83 & $1.34,2.50$ & 0.0001 \\
Rice intake $1.5-3.4 /$ wk vs. $<1.5$ times/wk & 1.07 & $0.80,1.44$ & 0.65 \\
Rice intake $\geq 3.5$ vs. $<1.5$ times/wk & 1.68 & $1.09,2.59$ & 0.02 \\
\hline
\end{tabular}

a Participants with "high" vs. "low" exposure patterns were clustered by k-means clustering method.

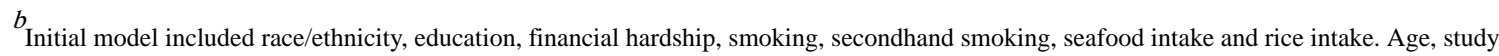
sites, and total energy intake, were forced in model selection.

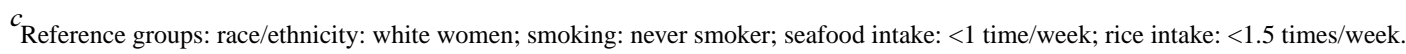

${ }^{d}{ }_{P}<0.05$ for all selected variables in backward elimination. 